\title{
Effect of Inclusion of Hepatitis B Vaccine in Childhood Immunization Program in India: A Retrospective Cohort Study
}

\author{
Rakesh Aggarwal, JJ Babu,*R Hemalatha,*Anumulu Venkateshar Reddy, *Divyanshu Sharma and \\ TARUN KUMAR \\ From the Department of Gastroenterology, Sanjay Gandhi Postgraduate Institute of Medical Sciences, Lucknow; and \\ *National Institute of Nutrition, Hyderabad, India. \\ Correspondence to: Dr Rakesh Aggarwal, Department of Gastroenterology, Sanjay Gandhi Postgraduate Institute of Medical Sciences, \\ Lucknow 226 014, India. \\ Received: January 31, 2014; Initial review: February 18, 2014; Accepted: September 15, 2014.
}

Objective: To assess the effectiveness of hepatitis B immunization program in a field setting in India.

Design: Serological survey of retrospective cohorts of children, vaccinated or not vaccinated with hepatitis $B$ vaccine.

Setting: Rural field areas of five districts in Andhra Pradesh state, where childhood hepatitis B immunization began in 2003.

Participants: Children aged 5-11 years who had received HB immunization ( $n=2674 ; 1357$ boys) or not received such immunization ( $n=2350 ; 1236$ boys).

Main Outcome Measures: Serum HBsAg, anti-HBc and antiHBs (quantitative) using automated enzyme-immunoassays in the year 2010.
Results: Anti-HBs positivity was higher among immunized than in unimmunized children (53\% vs.18\%; $P<0.001)$, and anti-HBc positivity was lower (1.1\% vs $10.8 \%$ : $P<0.01)$. HBsAg positivity was low in both the groups $(0.15 \%$ and $0.17 \% ; P=0.855)$. AntiHBs positivity rate declined with increasing age.

Conclusions: Administration of hepatitis B vaccine as part of Universal immunization program led to anti-HBs in a large proportion of children and a reduction in anti-HBc positivity, a marker of hepatatis $B$ virus infection. These data provide evidence supporting efficacy of hepatitis B immunization program in an Indian field setting, justifying the decision to include it in the universal immunization program.

Keywords: Evaluation, Impact, Program, Vaccination.
A dministration of hepatitis B vaccine to all newborns has been recommended in order to prevent chronic hepatitis B virus (HBV) infection, and the associated disease burden [14]. Inclusion of this vaccine in universal infant immunization programs in countries where HBV infection is highly endemic has led to a marked reduction in prevalence of chronic HBV infection, and in rate of occurrence of hepatocellular cancer [5,6]. In India, 2-4\% of healthy population has chronic HBV infection [7]. Government of India has included hepatitis B vaccine in the National universal immunization program in the entire country in 2011-12 [8]. Initially, a 3-dose schedule of 6, 10 and 14 weeks or of 0,6 and 14 weeks (if birth dose could be given) was used; this was later changed to 6,10 and 14 weeks with an additional dose being given within 24 hours of birth, where possible.

Effectiveness of $\mathrm{HB}$ vaccine in field situations is assessed using prevalence rates of: (i) hepatitis B surface antigen (HBsAg), an indicator of chronic HBV infection, (ii) anti-HBc, a marker of number of HBV infections, whether cleared or persistent, and (iii) anti-HBs, a protective antibody [9]. With successful vaccination, the prevalence of HBsAg and anti-HBc is expected to decline and that of anti-HBs to rise. The impact of infant immunization programs on $\mathrm{HBsAg}$ and anti-HBc prevalence is best measured when the immunized cohort reaches 5-7 years of age, since HBV infections acquired before this age have a high propensity to become chronic, and those acquired later rarely do so; thus majority of chronic HBV infections that could occur have accumulated by this age.

Accompanying Editorials: Pages 869-72.

Though data on coverage and drop-out rates of hepatitis B vaccine in the Indian setting are available [8], no data are yet available on the effectiveness of $\mathrm{HB}$ immunization in field settings. We therefore conducted this study in two retrospective cohorts of children, one consisting of those who had received hepatitis B immunization under field conditions and the other of those who had not.

\section{MeTHODS}

The study was conducted during 2010-11 in five districts of Andhra Pradesh (Rangareddy, Medak, Nizamabad, Karimnagar and Nalgonda) where hepatitis B immunization had been introduced in 2003-04. In each 
district, two mandals were identified randomly, and in each of these, 3-7 primary health centers and sub-centers were selected.

At each center or sub-center, two groups of children were enrolled: (a) those who had received three doses of hepatitis B vaccine as part of infant immunization program (mostly born in 2003 or 2004), and (b) those who had not received such immunization (mostly born in 2001 or 2002). The immunization status was verified using either immunization cards available with the parents or from records maintained at the primary health center/sub-center. Children whose immunization status could not be verified or documented, and those with partial immunization were excluded.

From each child, $3 \mathrm{~mL}$ of blood was drawn in a plain vial, and serum was separated, frozen and quickly transported to a laboratory where it was stored at $-80^{\circ} \mathrm{C}$ till analysis. The laboratory was not aware of the origin of individual specimens. Serum specimens were tested for HBsAg, total anti-HBc and anti-HBs using automated enzyme linked immunoassays (Roche Elecsys 411). The equipment automatically scored each test result as positive or negative based on the controls included in each kit, and calculated a titer for anti-HBs. To verify the test results, all the sera were retested for HBsAg and a subset were retested for anti-HBc and anti-HBs, using immunoassay kits from BioMerieux.

The required sample size for detection of difference in HBsAg prevalence rates in the immunized and unimmunized children was calculated using EpiInfo software and the following assumptions: baseline HBsAg rate among 5-year old children of 3\%, expected HBV carrier rate among immunized children of 1\%, acceptable alpha error of 0.05 , and study power of $90 \%$. The resulting numbers (1125 in each group) were corrected for cluster sampling using a 'design effect' of 2.0. Thus, it was proposed to study 2250 immunized and unimmunized children each.
Prevalence data in the two groups were compared using chi-square test. For anti-HBs titers, the raw data were converted into categories $(<10,10-100,101-1000$, and $>1000$ ) and compared using extended Mantel-Haenszel chi-square test for linear trend.

The study protocol was approved by Ethics committees of Sanjay Gandhi Postgraduate Institute of Medical Sciences, Lucknow and National Institute of Nutrition, Hyderabad. Permission was also obtained from health department of Andhra Pradesh and respective district health authorities, and both community (village level) and individual consents (from one of the parents) were obtained.

\section{RESULTS}

The study included 5024 children, of whom 2674 (1357 boys) had received hepatitis B immunization and 2350 (1236 boys) had not received it.

Table I shows the prevalence of HBsAg, anti-HBs and anti-HBc among immunized and unimmunized children. The positivity rate for anti-HBc, a marker of exposure to HBV infection (but not to hepatitis B vaccine), was lower among immunized children than among unimmunized children $(1.05 \%$ vs $1.79 \%$; risk ratio $=0.59[95 \%$ confidence interval $=0.36-0.94] ; P=0.026$ ). Among immunized children, a larger proportion tested positive for anti-HBs than unimmunized children (52.9\% vs. 17.7\%; $P<0.001$; RR $=2.98$ [95\% CI 2.71-3.28]), and mean antiHBs antibody titers were higher $(P<0.001)$ (Fig. 1).

In view of dissimilar age distributions of the immunized and unimmunized children, prevalence rates of anti-HBs and anti-HBc were compared in various age strata (Table II). Anti-HBs positivity rates in the immunized cohort showed a progressive decline $(P<0.001)$ with increasing age. In each age stratum, the prevalence of anti-HBs was higher among immunized children than in the unimmunized children; the difference was more marked at younger ages. The anti-HBc

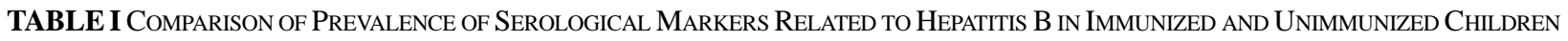

\begin{tabular}{|c|c|c|c|c|c|c|c|}
\hline \multirow[t]{2}{*}{ Serological marker } & \multicolumn{2}{|c|}{ Boys } & \multicolumn{2}{|l|}{ Girls } & \multicolumn{2}{|l|}{ All children } & \multirow[t]{2}{*}{ Pvalue } \\
\hline & $\begin{array}{c}\text { Immunized } \\
(n=1357)\end{array}$ & $\begin{array}{l}\text { Unimmunized } \\
\qquad(n=1236)\end{array}$ & $\begin{array}{c}\text { Immunized } \\
(n=1317)\end{array}$ & $\begin{array}{l}\text { Unimmunized } \\
\qquad(n=1114)\end{array}$ & $\begin{array}{c}\text { Immunized } \\
(n=2674)\end{array}$ & $\begin{array}{c}\text { Unimmunized } \\
\qquad(n=2350)\end{array}$ & \\
\hline Anti-HBs & $735(54 \%)$ & $233(19 \%)$ & $680(52 \%)$ & $184(17 \%)$ & $1415(52.9 \%)$ & $417(17.7 \%)$ & $<0.001$ \\
\hline Anti-HBc & $13(1.0 \%)$ & $28(2.3 \%)$ & $15(1.1 \%)$ & $14(1.3 \%)$ & $28(1.05 \%)$ & $42(1.79 \%)$ & 0.026 \\
\hline HBsAg & $3(0.2 \%)$ & $4(0.3 \%)$ & $1(0.1 \%)$ & $0(0 \%)$ & $4(0.15 \%)$ & $4(0.17 \%)$ & 0.855 \\
\hline
\end{tabular}

$P$ values relate to comparison of total immunized versus total unimmunized children. 


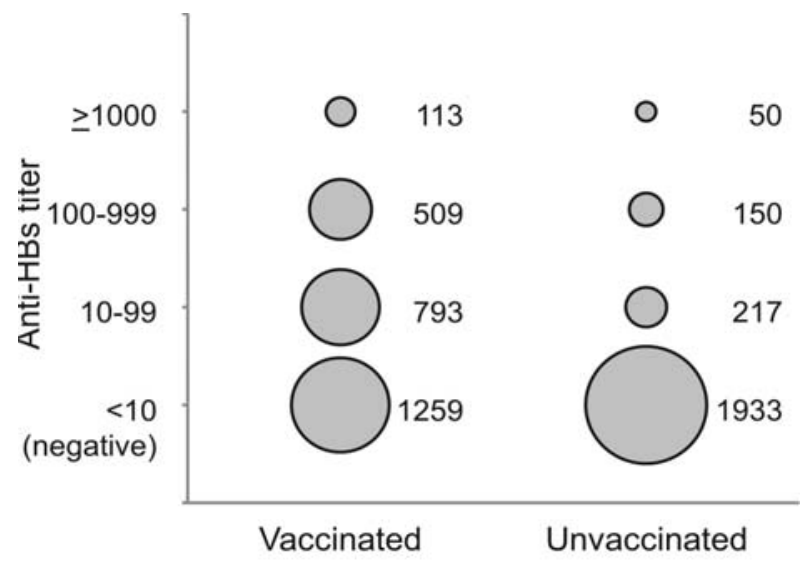

Fig. 1 Anti-HBs titers $(\mathrm{mIU} / \mathrm{mL})$ in immunized and unimmunized children. The area of each circle is proportional to the number of children with antibody titer in each range.

prevalence rates did not show significant differences between the immunized and unimmunized children in various age strata. Fig. 2 shows the relationship of antiHBs titers with age in the two cohorts. Among immunized children, the proportion of children with detectable antibody and those with higher titers declined with increasing age, indicating an age-related antibody decline.

Anti-HBs prevalence rates were similar among boys and girls (Table I), as were the anti-HBs titers (data not shown). The difference in anti-HBs positivity rate between immunized and unimmunized children was observed in each of the five districts (data not shown).

Retesting of 700 sera for anti-HBc and anti-HBs using kits from BioMerieux revealed results identical to those for the original testing. Retesting of all the 5024 sera for HBsAg using the BioMerieux kits showed positive test
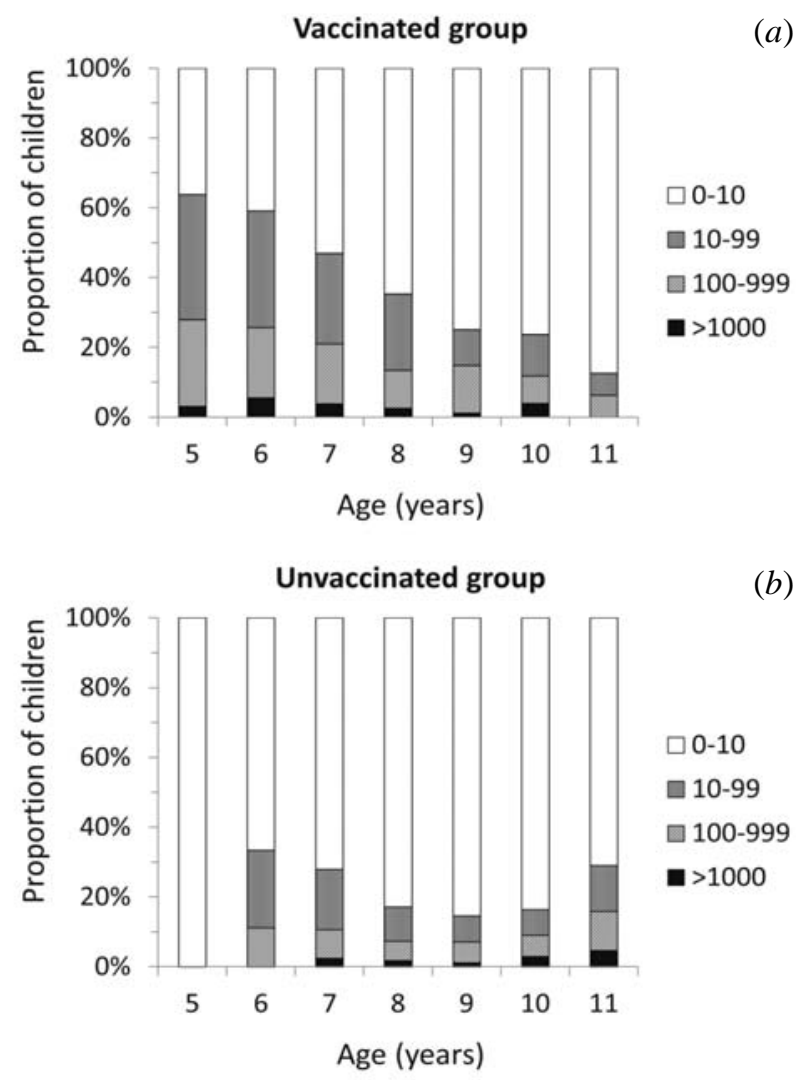

FIG. 2 Relationship of antibody titers ( $\mathrm{mIU} / \mathrm{mL}$ ) with age among immunized (a) and unimmunized (b) children.

results for 14 and borderline results for 10 specimens; the positive specimens included all the 8 specimens that had tested positive for HBsAg using the Roche kits. Of the 8 children who tested positive in both the assays, 7 were positive for anti-HBc. However, of the remaining 16 children who were positive $(n=6)$ or borderline $(n=10)$ on BioMerieux assay, only one was positive for anti-HBc.

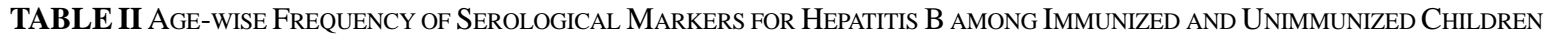

\begin{tabular}{llllll}
\hline \multirow{2}{*}{ Age (years) } & \multicolumn{2}{c}{ Anti-HBs } & & \multicolumn{2}{c}{ Anti-HBc } \\
\cline { 2 - 3 } \cline { 5 - 6 } & Immunized & Unimmunized & & Immunized & Unimmunized \\
\hline 4 & $0 / 1$ & - & & $0 / 1$ & $0 / 1$ \\
5 & $295 / 462(64 \%)$ & $0 / 1$ & $3 / 462(0.6 \%)$ & $0 / 9$ \\
6 & $676 / 1144(59 \%)$ & $3 / 9(33 \%)$ & & $11 / 1144(1.0 \%)$ & $4 / 208(1.9 \%)$ \\
7 & $360 / 768(47 \%)$ & $58 / 208(28 \%)$ & & $10 / 768(1.3 \%)$ & $13 / 619(2.1 \%)$ \\
8 & $42 / 119(35 \%)$ & $106 / 619(17 \%)$ & & $1 / 119(0.8 \%)$ & $6 / 643(0.9 \%)$ \\
9 & $22 / 88(25 \%)$ & $94 / 643(15 \%)$ & & $0 / 88$ & $15 / 763(2.0 \%)$ \\
10 & $18 / 76(24 \%)$ & $125 / 763(16 \%)$ & & $3 / 76(3.9 \%)$ & $4 / 107(3.7 \%)$ \\
\hline Total & $2 / 16(13 \%)$ & $31 / 107(29 \%)$ & & $0 / 16$ & $42 / 2350(1.8 \%)$ \\
\hline
\end{tabular}




\section{What is Already Known?}

- Universal hepatitis B immunization is useful in preventing HBV infection in populations with high endemicity for hepatitis B virus infection.

\section{What This Study Adds?}

- Inclusion of hepatitis B vaccine in Universal childhood immunization program of India has led to an increase in immunity against hepatitis B and protection against hepatitis B virus infection.

\section{Discussion}

In this retrospective cohort study, children who had received $\mathrm{HB}$ immunization as part of the universal immunization program in a field setting in Andhra Pradesh, India, showed a higher rate of anti-HBs positivity, and a reduced rate of anti-HBc positivity, than a cohort of children from the same area who had not received such immunization.

Presence of anti-HBs antibodies, which protect against HBV infection, can be related either to hepatitis B vaccination or to prior $\mathrm{HBV}$ infection. Their higher prevalence in the immunized cohort, despite a lower anti$\mathrm{HBc}$ seroprevalence in this group, indicates that the higher rate of anti-HBs in the immunized cohort was the effect of hepatitis B immunization. This implies that administration of hepatitis B vaccine in the Indian field setting did lead to induction of a protective antibody response against HBV. A significantly lower anti-HBc positivity rate in the immunized cohort in our study indicates that hepatitis B immunization in the Indian field setting was effective in reducing HBV transmission, thus justifying the decision to include this vaccine in the National immunization program.

Reduction in the anti-HBs seropositivity rate in the immunized cohort with increasing age is related to progressive decline in the titer of this antibody with time with its eventual disappearance in a subset of persons who have received hepatitis $B$ vaccine. Follow-up studies in several countries have shown that at 5-7 years after the completion of a 3-dose immunization schedule, only $60 \%$ to $85 \%$ of vaccinees had anti-HBs titer above the commonly-used cut-off of $10 \mathrm{mIU} / \mathrm{mL}$ [10-14]. Most of these studies were done in populations with high background rate of transmission of HBV. In India, an area with intermediate HBV endemicity, such decline of antiHBs response is expected to be more pronounced. However, despite the disappearance of anti-HBs, such immunized individuals continue to be protected against clinical illness as well as chronic HBV infection on exposure to this virus [15-17]. Thus, the proportion of children in the immunized cohort who are protected against HBV infection would be expected to be higher than the observed prevalence of anti-HBs antibody in this group.

Our study has some limitations. First, we failed to find a reduction in seroprevalence of HBsAg, a marker of chronic HBV infection, in the unimmunized cohort compared to the unimmunized cohort. There was a very low HBsAg seroprevalence in our cohort, as compared to earlier data from Andhra Pradesh [10]. This, along with a low anti-HBc positivity rate indicates that $\mathrm{HBV}$ transmission rate in the study population was much lower than our assumption, precluding detection of any difference in HBsAg positivity rates a that may have existed. This low transmission rate in our study area could be due to selection of a rural population for our study (whereas previous HBV seroprevalence studies are mainly from urban areas) [7], selection of villages with good immunization records (which may have had better health and medical care practices, and hence lower HBV transmission); and recent emphasis on safe injection practices in Andhra Pradesh. The anti-HBs positivity in our unimmunized cohort was also higher than expected. This was unlikely to be due to exposure to hepatitis B vaccine since anti-HBc rate in these children was low, and indicates a high rate of hepatitis B immunization outside the universal childhood immunization program in the unimmunized cohort.

It is important to use evidence to monitor and guide national immunization programs. Data from our study would be useful in this direction. Despite some limitations, our data indicate that hepatitis B immunization in field setting was effective in inducing protection against HBV infection and in reducing its transmission. Though we were unable to show a significant reduction in hepatitis B carrier rate, it would be useful to undertake similar studies in other parts of India where transmission of $\mathrm{HBV}$ is more frequent.

In conclusion, our study shows that hepatitis B immunization as part of Universal infant immunization program in the Indian population is effective in reducing the rate of HBV infection. These data should be useful to policy makers and health administrators to guide the national immunization program. 
Acknowledgement: Director, National Institute of Nutrition, Hyderabad.

Contributors: RA: study design, data acquisition, data analysis, writing the first draft and revising it critically; JJB: data acquisition, critical revision of the manuscript; RH: study design, data acquisition, critical revision of the manuscript; AVR and DS: data acquisition, critical revision of the manuscript; TK: data acquisition, drafting the manuscript, critical revision of the manuscript. All authors approved the final version.

Funding: Indian Council of Medical Research, New Delhi. Competing interests: None stated.

\section{REFERENCES}

1. McMahon BJ. Epidemiology and natural history of hepatitis B. Semin Liver Dis. 2005;25:3-8.

2. McMahon BJ, Alward WL, Hall DB, Heyward WL, Bender TR, Francis DP, et al. Acute hepatitis B virus infection: Relation of age to the clinical expression of disease and subsequent development of the carrier state. $\mathrm{J}$ Infect Dis. 1985;151:599-603.

3. World Health Assembly. Resolution WHA 45.17. Immunization and Vaccine Quality. Geneva: World Health Assembly, 1992.

4. World Health Organization. Hepatitis B vaccines: WHO position paper. Wkly Epidemiol Rec. 2009;84:405-19.

5. Chang MH, Chen CJ, Lai MS, Hsu HM, Wu TC, Kong MS, et al. Universal hepatitis B vaccination in Taiwan and the incidence of hepatocellular carcinoma in children. Taiwan Childhood Hepatoma Study Group. N Engl J Med. 1997;336:1855-9.

6. Liang X, Bi S, Yang W, Wang L, Cui G, Cui F, et al. Epidemiological serosurvey of Hepatitis B in China Declining HBV prevalence due to hepatitis B vaccination. Vaccine. 2009;27:6550-7.

7. Batham A, Narula D, Toteja T, Sreenivas V, Puliyel JM. Sytematic review and meta-analysis of prevalence of hepatitis B in India. Indian Pediatr. 2007;44:663-74.

8. Lahariya C, Subramanya BP, Sosler S. An assessment of hepatitis B introduction in India: Lessons for roll out and scale up of new vaccines in immunization programs. Indian J Public Hlth. 2013;57:8-14.

9. World Health Organization. Documenting the Impact of Hepatitis B Immunization: Best Practices for Conducting a
Serosurvey. Department of Immunization, Vaccines and Biologicals, World Health Organization, Geneva. 2011. WHO document WHO/IVB/11.08. Available from: www.who.int/ immunization/documents/who_ivb_11.08/ en/ý. Accessed April 23, 2014.

10. Wainwright RB, Bulkow LR, Parkinson AJ, Zanis C, McMahon BJ. Protection provided by hepatitis B vaccine in a Yupik Eskimo population. J Infect Dis. 1997;175:6747.

11. Lai CL, Wong CY, Yeoh EK, Lim WL, Chang WK, Lin HJ. Five-year follow-up of a prospective randomized trial of hepatitis B recombinant DNA yeast vaccine vs. plasma derived vaccine in children: immunogenicity and anamnestic responses. Hepatology. 1993;18:763-7.

12. Lee PI, Lee CY, Huang LM, Chang MH. Long-term efficacy of recombinant hepatitis $B$ vaccine and risk of natural infection in infants born to mothers with hepatitis B e antigen. J Pediatr. 1995;126:716-21.

13. Stevens CE, Toy PT, Taylor PE, Lee T, Yip HY. Prospects for control of hepatitis B virus infection: Implications of childhood vaccination and long-term protection. Pediatrics. 1992;90:170-3.

14. Da Villa G, Pelficcia MG, Peluso F, Ricciardi E, Sepe A. Anti-HBs responses in children vaccinated with different schedules of either plasma-derived or HBV DNA recombinant vaccine. Res Virol. 1997;148:109-14.

15. Banatvala J, Van Damme P, Oehen S. Lifelong protection against hepatitis $\mathrm{B}$ : the role of vaccine immunogenicity in immune memory. Vaccine. 2001;19:877-85.

16. European Consensus Group on Hepatitis B Immunity. Are booster immunisations needed for lifelong hepatitis B immunity? Lancet. 2000; 355:561-5.

17. Jack AD, Hall AJ, Maine N, Mendy M, Whittle HC. What level of hepatitis B antibody is protective? J Infect Dis. 1999;179:489-92.

18. Chandra M, Khaja MN, Farees N, Poduri CD, Hussain MM, Aejaz Habeeb M, et al. Prevalence, risk factors and genotype distribution of HCV and HBV infection in the tribal population: A community based study in south India. Trop Gastroenterol. 2003;24:193-5.

19. Patel DA, Gupta PA, Kinariwala DM, Shah HS, Trivedi GR, Vegad MM. An investigation of an outbreak of viral hepatitis B in Modasa town, Gujarat, India. J Glob Infect Dis. 2012;4:55-9. 